RESEARCH ARTICLE

\title{
High-energy, high-repetition-rate ultraviolet pulses from an efficiency-enhanced, frequency-tripled laser
}

\author{
Xinlin Lü ${ }^{1,2}$, Yujie Peng ${ }^{1}$, Wenyu Wang ${ }^{1,3}$, Yuanan Zhao ${ }^{4}$, Xiangyu Zhu ${ }^{4}$, and Yuxin Leng ${ }^{1}$ \\ ${ }^{1}$ State Key Laboratory of High Field Laser Physics and CAS Center for Excellence in Ultra-intense Laser Science, Shanghai Institute \\ of Optics and Fine Mechanics (SIOM), Chinese Academy of Sciences (CAS), Shanghai 201800, China \\ ${ }^{2}$ Center of Materials Science and Optoelectronics Engineering, University of Chinese Academy of Sciences, Beijing 100049, China \\ ${ }^{3}$ School of Optical and Electronic Information, Huazhong University of Science and Technology, Wuhan 430074, China \\ ${ }^{4}$ Laboratory of Thin Film Optics, Shanghai Institute of Optics and Fine Mechanics, Chinese Academy of Sciences, Shanghai 201800, \\ China
}

(Received 25 February 2021; revised 6 April 2021; accepted 9 April 2021)

\begin{abstract}
In this study, a high-energy, temporally shaped picosecond ultraviolet (UV) laser running at $100 \mathrm{~Hz}$ is demonstrated, with its pulses boosted to $120 \mathrm{~mJ}$ by cascaded regenerative and double-pass amplifiers, resulting in a gain of more than $10^{8}$. With precise manipulation and optimization, the amplified laser pulses were flat-top in the temporal and spatial domains to maintain high filling factors, which significantly improved the conversion efficiency of the subsequent third harmonic generation (THG). Finally, $91 \mathrm{~mJ}, 470$ ps pulses were obtained at $355 \mathrm{~nm}$, corresponding to a conversion efficiency as high as $76 \%$, which, as far as we are aware of, is the highest THG efficiency for a high-repetition-rate picosecond laser. In addition, the energy stability of the UV laser is better than $1.07 \%$ (root mean square), which makes this laser an attractive source for a variety of fields including laser conditioning and micro-fabrication.
\end{abstract}

Keywords: all-solid-state laser; third harmonic generation; ultraviolet laser

\section{Introduction}

High-power ultraviolet (UV) lasers are desirable for many applications, including laser conditioning, micro-nano fabrication, laser-propelled space debris removal, quantum optics, and nonlinear optical measurement ${ }^{[1-8]}$. Owing to limitations in the emission spectra of gain crystals, high-power UV lasers are usually obtained by third harmonic generation (THG) from near-infrared lasers. THG is a technique of nonlinear crystal-based sum frequency generation (SFG) between the fundamental and second harmonic frequencies; however, a consequence of two cascaded nonlinear processes is a low total conversion efficiency. The highest reported THG conversion efficiency from a picosecond solid-state laser is $80 \%$, which was based on a Nd-glass laser with a 20 min shot cycle and two $\mathrm{KH}_{2} \mathrm{PO}_{4}$ (KDP) crystals ${ }^{[9]}$. The THG conversion efficiency for high-repetition-rate sub-

Correspondence to: Y. Peng and Y. Leng, Shanghai Institute of Optics and Fine Mechanics (SIOM), Chinese Academy of Sciences (CAS), Shanghai 201800, China. Email: yjpeng@siom.ac.cn (Y. Peng); lengyuxin@mail.siom.ac.cn (Y. Leng) nanosecond and picosecond UV lasers is generally less than $50 \%$, which greatly limits their performance. In 2013, 12 ps, $39.1 \mathrm{~W}, 355 \mathrm{~nm}$ laser pulses were obtained using a multipass slab amplifier and $\mathrm{LiB}_{3} \mathrm{O}_{5}$ (LBO) crystals, which had a THG efficiency of $46 \%{ }^{[10]}$. In $2015,8 \mathrm{ps}, 234 \mathrm{~W}, 343 \mathrm{~nm}$ laser pulses were generated by a $300 \mathrm{kHz} \mathrm{Yb:YAG} \mathrm{thin-}$ disk laser and two LBO crystals with a THG conversion efficiency of $32 \%{ }^{[11]}$. In 2016, $37 \mathrm{ps}, 30.9 \mathrm{~W}, 300 \mathrm{kHz}$ laser pulses at $355 \mathrm{~nm}$ were obtained using $\mathrm{CsLiB}_{6} \mathrm{O}_{10}$ with a THG conversion efficiency of approximately $48 \%^{[12]}$. At a similar time, $1.13 \mathrm{~mJ}$ laser pulses at $355 \mathrm{~nm}$ with a pulse width of 40 ps were generated on the basis of one KDP crystal with a THG efficiency of $30.7 \%^{[13]}$. In 2020 , a conversion efficiency of $16.9 \%$ was achieved at $355 \mathrm{~nm}$ with a pulse duration of 568 ps using $\mathrm{LBO}$ and $\mathrm{Ca}_{5}\left(\mathrm{BO}_{3}\right)_{3} \mathrm{~F}$ crystals $^{[14]}$. Owing to a lack of precise temporal and spatial control, the actual power density of the above lasers differed from their average power density during the THG process and resulted in conversion efficiencies below their designed values. Therefore, it is desirable to develop a precise spatiotemporal manipulation technique to improve the filling factor 
of laser pulses and ensure the uniformity of the spatial and temporal power densities. This level of control would allow designed and actual THG efficiencies to be similar after optimization.

In this paper, we present a high-energy, high-repetitionrate, temporally shaped UV laser. Our system consists of a Nd:YAG-based fundamental-frequency laser and an efficiency-enhanced harmonic generation unit. The Nd:YAG laser is a diode-pumped solid-state laser (DPSSL), which achieves temporal programmable laser pulses with $120 \mathrm{~mJ}$ energy at $100 \mathrm{~Hz}$ repetition rate using a master oscillator power amplifier (MOPA). To this system, we added two cascaded LBO nonlinear crystals for THG. Through precise temporal and spatial shaping, we were able to achieve an extremely high THG efficiency of $76 \%$ at $91 \mathrm{~mJ}, 355 \mathrm{~nm}$ pulses with a duration of $470 \mathrm{ps}$ at full width at half maximum (FWHM). To the best of the authors' knowledge, this is the highest THG conversion efficiency for a highpower, high-repetition-rate picosecond solid-state laser.

\section{Experimental setup}

Figure 1 shows the schematic of our UV laser system. Temporal programmable laser pulses were generated from a commercial single longitudinal mode fiber laser with an arbitrary waveform generator (AWG), which allowed the output wavelength to be accurately tuned by changing the temperature of the distributed feedback seed laser to suppress amplified spontaneous emission effectively during the subsequent amplification process, where the fiber laser supplies $\sim 1 \mathrm{~nJ}, 500 \mathrm{ps}$ seed pulses at a repetition rate of $100 \mathrm{~Hz}$ and a wavelength of $1064.2 \mathrm{~nm}$. After passing through a fiber isolator, the seed laser beam is collimated by an aspheric lens and passes through an optical isolator, which is composed of a polarization beam splitter (PBS), Faraday rotator (FR), and half-wave plate (HWP). Two thin- film plates (TFPs) were also added to control the polarization direction of the injected and output laser pulses from the regenerative amplifier (RA). A Pockels cell (PC) in the RA was operated at a quarter-wave voltage and uses a quarterwave plate (QWP) to maintain laser pulses at p-polarization during round trips. Laser diode (LD) module 1 is sidepumped and contains a $\mathrm{Nd}$ :YAG rod, $3 \mathrm{~mm}$ in diameter and $63 \mathrm{~mm}$ in length, which, when pumped with $250 \mu \mathrm{s}$ pulses, provides a small signal gain of 1.8. When the quarter-wave voltage is applied to the PC, the pulses are amplified in the RA cavity every time they pass through LD module 1 . After several round trips, a pulse with a total energy of $6.5 \mathrm{~mJ}$ and beam diameter of $1.5 \mathrm{~mm}$ is delivered from the RA, and the energy stability within $3 \mathrm{~h}$ of the laser pulses is $\sim 0.68 \%$ (root mean square (RMS)).

After the optical isolator, the laser pulse is injected into a beam shaper and amplified by a double-pass amplifier. The beam shaper consists of a beam expander with a planoconcave lens and a plano-convex lens that expand the Gaussian beam diameter to $12 \mathrm{~mm}$. To achieve a high spatial filling factor, we used a specially designed $8 \mathrm{~mm}$ diameter soft-edge aperture that shapes the beam profile to a flattop distribution, which matches the optical aperture of the amplification module in the double-pass amplifier. Beam quality was ensured using three image-relay Kepler telescopes, which consist of a $4 f$-based optical system with two plano-convex lenses to maintain a flat-top beam distribution after propagation with a 1:1 expansion ratio. The object plane of the $4 f$ image-relay setup is at the soft-edge aperture, and the image planes are at the center of LD module 2, at the end mirror of the double-pass amplifier, and between the two nonlinear crystals in the harmonic generation unit, respectively. The beam shaping ensures that the amplified beam has a flat-top distribution for high-efficiency harmonic generation. After the beam shaping unit, the pulse energy is reduced to $3 \mathrm{~mJ}$.

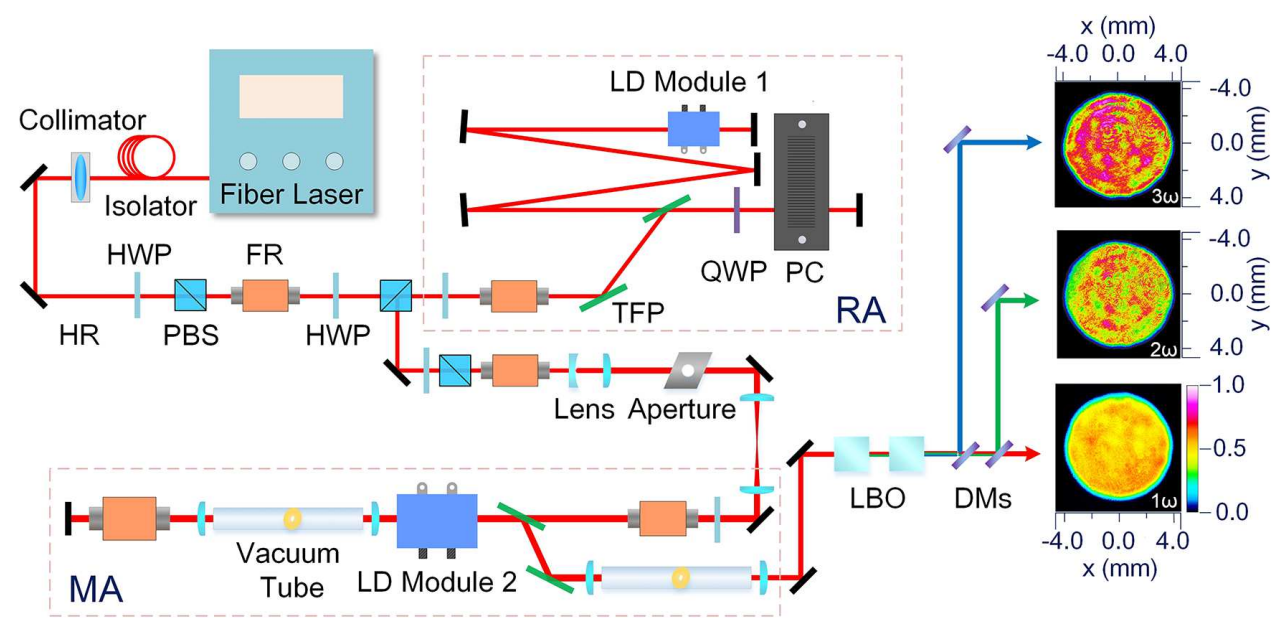

Figure 1. Schematic of the UV laser system and transverse beam profiles of the amplified pulses at $1064 \mathrm{~nm}(\omega), 532 \mathrm{~nm}(2 \omega)$, and $355 \mathrm{~nm}(3 \omega)$ measured at their maximum energies via relay imaging. RA, regenerative amplifier; MA, main amplifier; HR, high reflector; HWP, half-wave plate; PBS, polarization beam splitter; FR, Faraday rotator; TFP, thin-film plate; QWP, quarter-wave plate; PC, Pockels cell; LD, laser diode; DM, dichroic mirror. 
In the double-pass main amplifier (MA), LD module 2 with a $10 \mathrm{~mm}$ aperture can provide a small signal gain of 10 when operating at $100 \mathrm{~Hz}$. However, as the Nd:YAG rod is side-pumped, the homogeneity of the amplification is dependent on the absorbance of the pumping light by Nd:YAG ${ }^{[15]}$. To counter this, the five packaged LD submodules were distributed asymmetrically to ensure uniform pumping, and a circulatory water-cooling system was installed to maintain a steady LD module temperature and remove redundant heat to prevent fracturing of the gain crystal. The temperature of the water-cooling circulatory system was set to be stabilized at $27.5^{\circ} \mathrm{C}$, which benefits the $\mathrm{LD}$ emission wavelength to match the absorption peak of the Nd:YAG crystal at $808 \mathrm{~nm}$. The combined effect of the LD pumping and water-cooling systems was a non-uniform radial temperature distribution in the rod-shaped crystals, resulting in the Nd:YAG rod acting as a lens. To compensate for this thermal lensing effect, the second image-relay system was designed precisely so that the distance between the two plano-convex lenses was closer than what would normally be required. To prevent the laser pulses from ionizing the air at the focus, they are directed through a vacuum tube in the image-relay setup; furthermore, a pinhole set at the focal plane filtered the spatial high-frequency components to further maintain a flat-top beam distribution for the amplified beams. Thermal stress in the rod-shaped crystals resulted in a refractive index change because of the photoelastic effect, which led to thermally induced birefringence ${ }^{[16]}$. This birefringence can lead to depolarization and distortion of the output beam profile after passing through polarization-related devices ${ }^{[17]}$. When the laser beam travels through the same LD module and rod twice with a $90^{\circ}$ polarization rotation, the radial and tangential polarizations also travel the same optical path length ${ }^{[18]}$. To mitigate this effect, an FR was introduced to the double-pass configuration to achieve the polarization rotation while compensating for the thermal birefringence effect, resulting in laser beams that exited the TFP with flattop near-field spatial profiles, as shown in Figure $1(1 \omega)$. The energies of the laser pulses were boosted to 25 and $120 \mathrm{~mJ}$ after single- or double-passing LD module 2, respectively.

After passing through the third image-relay setup, the amplified pulses from the MA were injected into the harmonic generation unit, where two cascaded LBO crystals were employed to achieve THG. Both crystals were placed in constant temperature ovens to maintain stable temperature matching, and angular phase matching was achieved by their accurate alignment. The first LBO has an aperture of $12 \mathrm{~mm}$ $\times 12 \mathrm{~mm}$ and length of $10 \mathrm{~mm}$ and was cut at $\theta=90^{\circ}$ with $\varphi=11.3^{\circ}$ for a type-I phase match. Up to $99.6 \mathrm{~mJ}$, $532 \mathrm{~nm}$ laser pulses were obtained with $120 \mathrm{~mJ}$ injected laser energy at $1064 \mathrm{~nm}$. Figure $1(2 \omega)$ shows the nearfield beam distribution of $532 \mathrm{~nm}$ laser pulses with $99.6 \mathrm{~mJ}$ energy. The second LBO crystal has a dimension of $12 \mathrm{~mm}$ $\times 12 \mathrm{~mm} \times 15 \mathrm{~mm}$ and was cut at $\theta=42.5^{\circ}$ with $\varphi$ $=90^{\circ}$ for type-II phase matching. The spatial separation distance of these two LBO crystals is approximately $25 \mathrm{~mm}$ and focal lengths of the plano-convex lenses in the third Kepler telescope are both $280 \mathrm{~mm}$. The energy ratio of the fundamental and the second harmonic laser pulses is approximately $1: 2$ for highly efficient frequency tripling, and the peak intensities of the $1064 \mathrm{~nm}$ and $532 \mathrm{~nm}$ beams are about 169.4 and $338.8 \mathrm{MW} / \mathrm{cm}^{2}$, respectively. Consequently, laser pulses at $355 \mathrm{~nm}$ were generated by SFG in the second LBO crystal and achieved an approximate flat-top nearfield beam distribution at the maximum energy with spatial optimization, as shown in Figure $1(3 \omega)$.

\section{Results and discussion}

Owing to the gain saturation effect of the Nd:YAG amplifier, the temporal width of the laser pulses was shortened after passing the MA; furthermore, because of temporal distortion during the amplification process, the temporal power density of the front edge was higher than that of the back edge of the output laser pulses, which resulted in a low filling factor and low THG conversion efficiency. Figures 2(a) and 2(b) show the time-domain waveforms of an unmodulated $1064 \mathrm{~nm}$ seed laser and the corresponding $355 \mathrm{~nm}$ laser, where the THG efficiency, without temporal optimization, was approximately $60 \%$, which can be further improved by programming seed lasers in the time domain with the AWG. Because the length of the LBO crystal was designed to work at the average power density of laser pulses, high beam filling factors reduced the power density difference between each point of the laser beam and the corresponding average value; therefore, the peak power density of each point was at or close to the optimal value for THG, and a high triple frequency efficiency was achieved with a high spatial filling factor. In addition, the temporal distribution with a high filling factor is also the optimal distribution for high THG efficiency. To obtain amplified pulses with a high temporal filling factor, the fiber laser AWG was used to modulate the pulse duration and temporal shape of the seed laser. After optimizing the injection laser to have the same average amplified power, the amplified pulses were temporally flat-top to maintain a high filling factor, and modulated injection compensated for the temporal distortion of the laser pulses following amplification and THG, resulting in a temporal waveform output with a high $355 \mathrm{~nm}$ filling factor, as depicted in Figures 2(c) and 2(d). The resulting THG conversion efficiency from this temporal optimization was $76 \%$ from 1064 to $355 \mathrm{~nm}$, which verified that a high filling factor is an important factor in this optimization process.

We measured the energy of $355 \mathrm{~nm}$ output pulses under seven different $1064 \mathrm{~nm}$ pulse width conditions, and Figure 3 shows the corresponding relationships between the THG conversion efficiency and measured $355 \mathrm{~nm}$ output pulse widths (FWHM). For each pulse width condition, the energy 

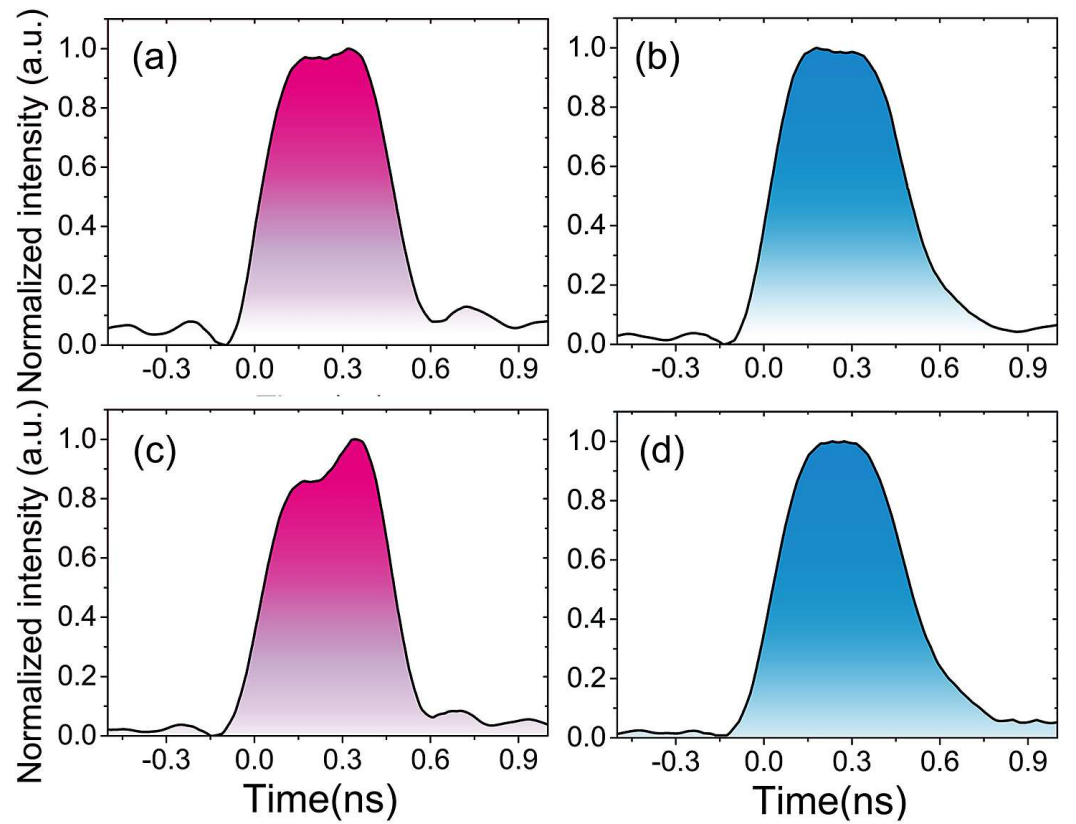

Figure 2. Time-domain waveforms of (a) an unmodulated $1064 \mathrm{~nm}$ seed laser pulse, (b) its corresponding $355 \mathrm{~nm}$ output, (c) a programmed $1064 \mathrm{~nm}$ seed laser pulse, and (d) its corresponding $355 \mathrm{~nm}$ output.

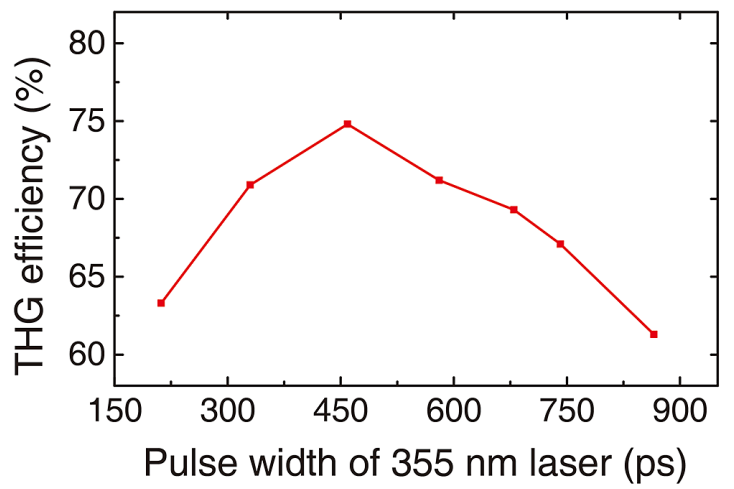

Figure 3. Relationship between THG conversion efficiency and the measured pulse width (FWHM) of the $355 \mathrm{~nm}$ output laser.

of the $1064 \mathrm{~nm}$ laser was also changed to maintain the same peak power density for THG. In addition, each seed laser injection was optimized to ensure that the $355 \mathrm{~nm}$ output retained a spatial and temporal flat-top distribution to keep a high filling factor for THG. As can be seen in Figure 3, the maximum THG conversion efficiency is $76 \%$ when the pulse width of the $355 \mathrm{~nm}$ laser was approximately $470 \mathrm{ps}$ and the pulse width of the corresponding $1064 \mathrm{~nm}$ seed laser with spatial and temporal optimization was $500 \mathrm{ps}$.

In order to evaluate the far-field beam quality of the UV laser after spatial and temporal optimization, encircled energy function of the two-dimensional integral for the diffraction-limit was deduced ${ }^{[19,20]}$; however, the chargecoupled device (CCD) employed for beam diagnostics was a square pixel-type detector, so ensquared rather than encircled energy was utilized ${ }^{[21]}$. Figure 4(a) shows the ideal ensquared energy of a focused flat-top beam and the actually measured ensquared energy of far-field laser beams, and Figures 4(b)4(d) show the corresponding far-field beam profiles of 1064 , 532, and $355 \mathrm{~nm}$ pulses measured at their maximum energies, respectively. These data demonstrate that the wavefront of the laser pulses distorts and the beam quality degrades a little during the nonlinear process. Spatial manipulation was effective at maintaining an approximate flat-top distribution for near-field $355 \mathrm{~nm}$ output beam profiles to achieve highefficiency THG. In terms of the astigmatism, the far-field $355 \mathrm{~nm}$ output beam profile was close to that of the $1064 \mathrm{~nm}$ output with the vertical and horizontal directions in sharp focus at almost equal distances after spatial and temporal optimization.

To improve the energy stability of the $355 \mathrm{~nm}$ output laser pulses, we controlled the stabilities of the time-domain waveforms of laser pulses and temperatures of the two LBO crystals. When the DPSSL system was operated at a wavelength of $1064 \mathrm{~nm}$ and $100 \mathrm{~Hz}$ repetition rate with a $120 \mathrm{~mJ}$ pulse energy and $500 \mathrm{ps}$ duration (FWHM), the energy stability within $3 \mathrm{~h}$ was $0.82 \%$ (RMS), as can be seen in Figure 5. In addition, following spatial and temporal optimization, laser pulses at a wavelength of $355 \mathrm{~nm}$ and $470 \mathrm{ps}$ width (FWHM) were generated with $91 \mathrm{~mJ}$ energy, and the energy stability in $3 \mathrm{~h}$ was $1.07 \%$ (RMS), as shown in Figure 5. The temperature stability relaxation of the LBO crystals also caused the periodic fluctuation of the energy stability of the $355 \mathrm{~nm}$ lasers. 


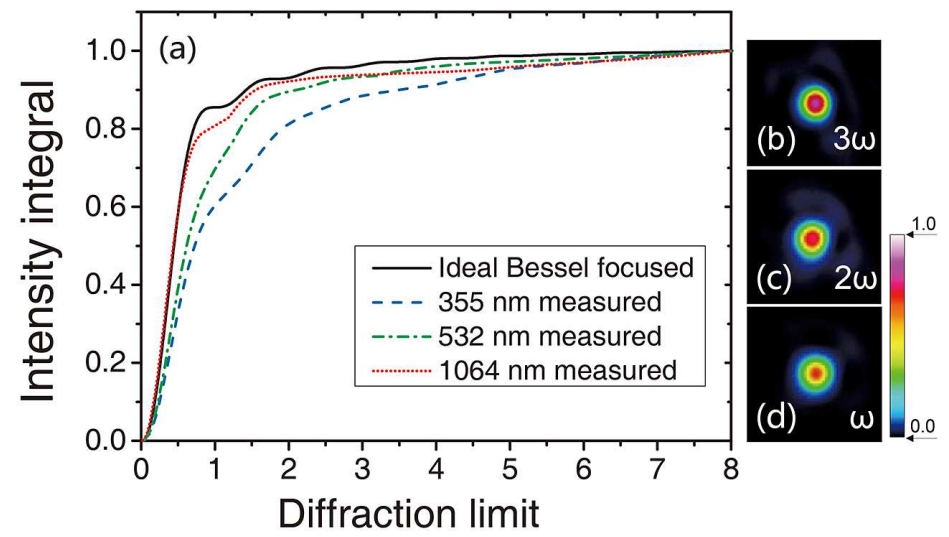

Figure 4. (a) Ideal ensquared energy of a focused flat-top beam and the actually measured ensquared energy of various far-field laser beams: (b) $355 \mathrm{~nm}$, (c) $532 \mathrm{~nm}$, and (d) $1064 \mathrm{~nm}$ measured at their maximum energies.

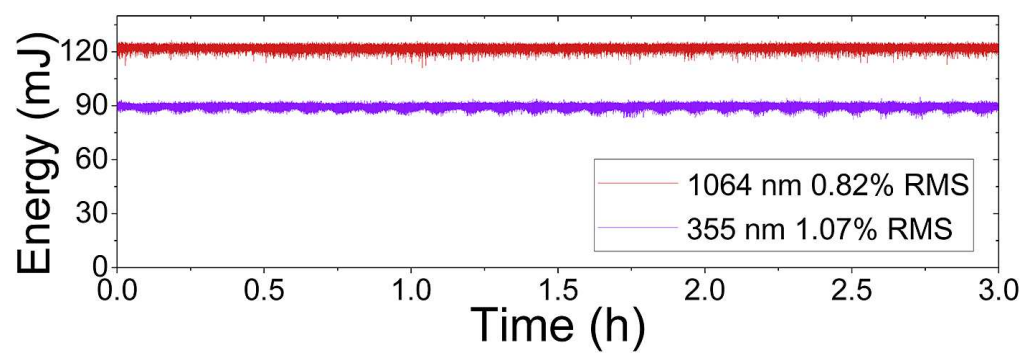

Figure 5. Energy stability of $1064 \mathrm{~nm}$ and $355 \mathrm{~nm}$ laser pulses.

\section{Conclusions}

In summary, using spatial and temporal manipulation techniques, we have demonstrated a high-energy, high-repetitionrate stabilized picosecond UV laser with an enhanced THG conversion efficiency, which delivered $91 \mathrm{~mJ}$ pulses with a duration of $470 \mathrm{ps}$ at a frequency of $100 \mathrm{~Hz}$. The $355 \mathrm{~nm}$ laser pulses were generated from a $120 \mathrm{~mJ} 1064 \mathrm{~nm}$ picosecond MOPA-based DPSSL and subsequent LBO crystalbased THG system. The $1064 \mathrm{~nm}$ DPSSL featured a flattop spatial and temporal distribution and $0.82 \%$ (RMS) energy stability within $3 \mathrm{~h}$. Spatial modulation devices, including a soft-edge aperture, image-relay setup, FR, and spatial filter, were used to maintain a high spatial filling and correspondingly high THG conversion efficiency. Consequently, spatial gain distortion, thermal lensing, and depolarization effects were effectively compensated for, which resulted in a high-power laser beam output with a flattop spatial distribution. Moreover, through high-precision temporal waveform modulation and accurate phase matching to improve the temporal filling factor of the UV laser, we were able to achieve an extremely high THG efficiency of $76 \%$ with a $355 \mathrm{~nm}$ pulse width of $470 \mathrm{ps}$ (FWHM). To the best of the authors' knowledge, this is the highest THG conversion efficiency in a high-power, high-repetition-rate picosecond solid-state laser. In addition, following spatial and temporal optimization, the far-field $355 \mathrm{~nm}$ beam output profile was close to that of the $1064 \mathrm{~nm}$ beam, and the energy stability within $3 \mathrm{~h}$ of the $355 \mathrm{~nm}$ pulses was $1.07 \%$ (RMS) operating at $91 \mathrm{~mJ}$. We consider this high-energy, high-repetition-rate picosecond UV pumping laser system to be an attractive pumping source for a wide variety of applications.

\section{Acknowledgments}

This work was supported by the National Natural Science Foundation of China (61925507 and 62075227), National Key R\&D Program of China (2017YFE0123700), Strategic Priority Research Program of the Chinese Academy of Sciences (XDB1603), Program of Shanghai Academic/Technology Research Leader (18XD1404200), Shanghai Municipal Science and Technology Major Project (2017SHZDZX02), and Youth Innovation Promotion Association CAS (2020248).

\section{References}

1. F. Köttig, F. Tani, C. M. Biersach, J. C. Travers, and P. S. J. Russell, Optica 4, 1272 (2017).

2. D. Chen, B. Wang, H. Wang, X. Zhu, Z. Xu, Y. Zhao, S. Wang, K. Ni, L. Zheng, H. Zhang, H. Qi, and J. Shao, High Power Laser Sci. Eng. 8, e6 (2020).

3. J. A. Jarboe, G. J. Exarhos, J. J. Adams, A. H. Guenther, K. L. Lewis, R. P. Hackel, D. Ristau, M. J. Soileau, and C. J. Stolz, Proc. SPIE 6720, 67200J (2007). 
4. M. K. Kelly, O. Ambacher, B. Dahlheimer, G. Groos, R. Dimitrov, H. Angerer, and M. Stutzmann, Appl. Phys. Lett. 69, 1749 (1996).

5. E. Y. Loktionov and M. M. Skobelev, J. Phys. Conf. Ser. 1147, 012074 (2019).

6. J. Yin, J. G. Ren, H. Lu, Y. Cao, H. L. Yong, Y. P. Wu, C. Liu, S. K. Liao, F. Zhou, Y. Jiang, X. D. Cai, P. Xu, G. S. Pan, J. J. Jia, Y. M. Huang, H. Yin, J. Y. Wang, Y. A. Chen, C. Z. Peng, and J. W. Pan, Nature 488, 185 (2012).

7. Y. Li, C. Wang, Y. Zhang, P. Hu, S. Zhang, M. Du, X. Su, Q. Li, and F. Yun, Photonics Res. 8, 806 (2020).

8. M. Wang, F. Ma, Z. Wang, D. Hu, X. Xu, and X. Hao, Photonics Res. 6, 307 (2018).

9. W. Seka, S. D. Jacobs, J. E. Rizzo, R. Boni, and R. S. Craxton, Opt. Commun. 34, 469 (1980).

10. P. Zhu, D. Li, Q. Liu, J. Chen, S. Fu, P. Shi, K. Du, and P. Loosen, Opt. Lett. 38, 4716 (2013).

11. J. P. Negel, A. Loescher, A. Voss, D. Bauer, D. Sutter, A. Killi, M. A. Ahmed, and T. Graf, Opt. Express 23, 21064 (2015).
12. K. Ueda, Y. Orii, Y. Takahashi, G. Okada, Y. Mori, and M. Yoshimura, Opt. Express 24, 30465 (2016).

13. H. Qi, Z. Wang, F. Yu, X. Sun, X. Xu, and X. Zhao, Opt. Lett. 41, 5823 (2016).

14. F. Cassouret, A. Kausas, V. Yahia, G. Aka, P. Loiseau, and T. Taira, Opt. Express 28, 10524 (2020).

15. D. W. Noom, S. Witte, J. Morgenweg, R. K. Altmann, and K. S. Eikema, Opt. Lett. 38, 3021 (2013).

16. H. Su, Y. Peng, J. Chen, Y. Li, P. Wang, and Y. Leng, Appl. Sci. 7, 997 (2017).

17. W. Koechner, in Solid-State Laser Engineering, W. Koechner (ed.) (Springer New York, New York, 2006), p. 423.

18. Q. Lü, N. Kugler, H. Weber, S. Dong, N. Müller, and U. Wittrock, Opt. Quant. Electron. 28, 57 (1996).

19. A. M. Clements and J. E. Wilkins, J. Opt. Soc. Am. 64, 23 (1974).

20. J. J. Stamnes, H. Heier, and S. Ljunggren, Appl. Opt. 21, 1628 (1982).

21. T. B. Andersen, Appl. Opt. 54, 7525 (2015). 